\title{
Multimorbidity: can general practitioners identify the health conditions most important to their patients? Results from a national cross-sectional study in Switzerland
}

Anouk Déruaz-Luyet ${ }^{1}$, Alexandra A. N'Goran ${ }^{1}$, Jérôme Pasquier², Bernard Burnand² ${ }^{2}$ Patrick Bodenmann ${ }^{3}$, Stefan Zechmann ${ }^{4}$, Stefan Neuner-Jehle ${ }^{4}$, Nicolas Senn ${ }^{1}$, Daniel Widmer ${ }^{1}$, Sven Streit ${ }^{5}$, Andreas Zeller ${ }^{6}$, Dagmar M. Haller ${ }^{7}$ and Lilli Herzig ${ }^{1^{*}}$ (D)

\begin{abstract}
Background: Faced with patients suffering from more than one chronic condition, or multimorbidity, general practitioners (GPs) must establish diagnostic and treatment priorities. Patients also set their own priorities to handle the everyday burdens associated with their multimorbidity and these may be different from the priorities established by their GP. A shared patient-GP agenda, driven by knowledge of each other's priorities, would seem central to managing patients with multimorbidity. We evaluated GPs' ability to identify the health condition most important to their patients.

Methods: Data on 888 patients were collected as part of a cross-sectional Swiss study on multimorbidity in family medicine. For the main analyses on patients-GP agreement, data from 572 of these patients could be included. GPs were asked to identify the two conditions which their patient considered most important, and we tested whether either of them agreed with the condition mentioned as most important by the patient. In the main analysis, we studied the agreement rate between GPs and patients by grouping items medicallyrelated into 46 groups of conditions. Socio-demographic and clinical variables were fitted into univariate and multivariate models.
\end{abstract}

Results: In 54.9\% of cases, GPs were able to identify the health condition most important to the patient. In the multivariate model, the only variable significantly associated with patient-GP agreement was the number of chronic conditions: the higher the number of conditions, the less likely the agreement.

Conclusion: GPs were able to correctly identify the health condition most important to their patients in half of the cases. It therefore seems important that GPs learn how to better adapt treatment targets and priorities by taking patients' perspectives into account.

Keywords: Multimorbidity, Family medicine, Patient-provider agreement

\footnotetext{
* Correspondence: Lilli.herzig@hin.ch

${ }^{1}$ Institute of Family Medicine, University of Lausanne, 44 rue du Bugnon,

1011 Lausanne, Switzerland

Full list of author information is available at the end of the article
}

(c) The Author(s). 2018 Open Access This article is distributed under the terms of the Creative Commons Attribution 4.0 International License (http://creativecommons.org/licenses/by/4.0/), which permits unrestricted use, distribution, and reproduction in any medium, provided you give appropriate credit to the original author(s) and the source, provide a link to the Creative Commons license, and indicate if changes were made. The Creative Commons Public Domain Dedication waiver (http://creativecommons.org/publicdomain/zero/1.0/) applies to the data made available in this article, unless otherwise stated. 


\section{Background}

Multimorbidity is defined as the co-occurrence of two (or three) or more chronic health conditions in one person without any condition being considered an index condition [1]. Faced with patients with multimorbidity, GPs must often set priorities between the different chronic conditions, as managing them all is often infeasible in everyday practice. Patients also set their own disease and treatment priorities to handle the everyday burdens associated with their multimorbidity and these may be different from the priorities established by their GP.

Evidence shows that good communication between patients and GPs leads to more agreement between them and a better care outcome [2]. However, studies examining the match between physicians' and patients' priority health conditions have produced contrasting results, and most of these studies were conducted in diabetic patients [3-5]. High levels of agreement on which conditions were considered important from patients' and GPs' perspectives have been reported previously, but levels of agreement were lower in patients reporting a poor health status [5]. A common patient-GP (or patientprovider) agenda is key to a successful therapeutic process, particularly in patients with multimorbidity, and this is generally dependent on good patient-GP communication and shared decision-making [2, 6, 7]. Our hypothesis was that more experienced GPs and GPs with a sub-specialization in psychosocial and psychosomatic medicine, based on the development of strong communication, would be positively associated with agreement between GPs and patients, as would a higher number of visits to the GP in the last 12 months and more years of follow-up by the same GP. In contrast, a high number of chronic conditions and a greater number of medical doctors involved in the patient's treatment could be a reflection of more complicated cases or sicker patients, for whom setting priorities would be more difficult. Lower health-literacy and less well-educated patients could also be factors complicating patient-GP communication and could be negatively associated with agreement rates between GPs and patients. The presumed direction of the association between the number of consultations handled by the GP each week was less clear-cut, however, as more consultations per week could mean either greater knowledge of each patient with multimorbidity or less time spent per consultation.

Studies to date in primary care settings have reported low levels of agreement between patients and GPs on health and treatment priorities [8]. Qualitative studies have underlined that they do not seem to give the same level importance to the same conditions, and to some extent, GPs appear unaware of their patients' priorities [8-10]. Voigt et al. [8] showed that even if GPs were able to identify the number of health problems that their patients considered important, they did not give the same level of importance to the same conditions. Patients' ratings of health conditions have been shown to be related to their current experience of their condition, whereas GPs' ratings were more related to the condition's prognosis [9]. Thus, our aim was to evaluate whether GPs could identify the condition that their patients with multimorbidity considered most important. To the best of our knowledge, this is one of the first quantitative study addressing this topic.

\section{Methods}

We analyzed data from the Multimorbidity in Family Medicine study, a cross-sectional study in Switzerland. The study protocol and a detailed description of the database established have been published elsewhere $[11,12]$.

\section{Setting and participants}

Data collection took place in Switzerland between January and September 2015. A convenience sample of 100 GPs randomly enrolled patients during scheduled consultations at their private practices. Each GP was provided with a randomisation calendar specifying which patients to enrol on each half-day during the recruitment weeks. All multimorbid patients above 18 years old, followed by their GP for at least 6 months and suffering from at least three of the 75 chronic conditions on a predefined list based on the International Classification of Primary Care 2 (ICPC 2) were considered eligible $[13,14]$ and signed a written consent to participate to the study. GPs completed a paper-based questionnaire for each included patient and patients enrolled completed a telephone-based questionnaire. Only anonymous dates were transmitted to the research team. The study protocol ( $\left.n^{\circ} 314 / 15\right)$ was approved by the Human Research Ethics Committee of the Canton Vaud.

\section{Variables \\ Outcome variable}

We evaluated the agreement between what patients considered to be their most important health condition and what GPs thought patients considered to be their most important health condition. Conditions were considered to be "in agreement" if one of two conditions considered important by the GP belonged to the same group of conditions as the one considered most important by the patient; they were "in disagreement" otherwise. Agreement between GPs and patients was considered as a dichotomous variable.

\section{Independent variables}

The GP-related variables of interest were: sex, number of years of practice, number of consultations per week, practice location, and a sub-specialization in psychosocial and 
psychosomatic medicine, i.e., a postgraduate qualification to handle psychosomatic and psychosocial factors influencing the development and management of diseases. The patient-related variables were: age, sex, number of chronic conditions, level of education, number of medical doctors involved in the patient's treatment, number of visits to the GP in the last 12 months, and number of years with this GP.

\section{Data sources GP level}

We asked the GPs to list the two health conditions they thought were most important to their patients.

\section{Patient level}

During a telephone interview, patients were asked, "If you had the power to heal one of your medical problems, which one would you choose first?" In the absence of a validated question on this topic, we designed this question during several internal consensus discussions. Although it did not precisely match the GPs' question, it could be answered by patients with lower levels of health literacy. In line with the study's protocol and its overall goals, interviewers were trained to conduct standardized telephone interviews. As a result, they did not to attempt to establish more precise patient answers when they might have been needed.

\section{Measurement}

Only answers that could be transferred into a corresponding ICPC-2 code were used. In the knowledge that patients and GPs may refer to the same conditions differently and with different levels of precision, we grouped related chronic conditions for agreement analyses. For the main analysis, we considered a classification involving 46 groups of diseases derived from the classification developed by van den Bussche et al. for elderly patients with multimorbidity and subsequently adapted specifically to our assessment by three members of the research team [15]. (Additional file 1: Table S1). We evaluated agreement between GPs' and patients' answers based on these 46 groups. To avoid overinterpretation, for both analyses, any responses that could not be unequivocally attributed to one group were considered as missing data. In a sensitivity analysis, we used fifteen chapters of the ICPC-2 classification to group items (General and unspecified; Blood/bloodforming organs and immune system; Digestive; Eye; Ear; Cardiovascular; Musculoskeletal; Neurological; Psychological; Respiratory; Skin; Endocrine/metabolic and nutritional; Urological; Female Genital; Male Genital). We excluded the two chapters on pregnancy and social aspects, which did not encompass chronic conditions per se [16]. We also considered answers in which more than one code was given, as follows. If all of the codes belonged to the same group or chapter, we attributed the condition to that group or chapter, respectively. If the codes did not belong to the same group or chapter, or if the group or chapter could not be clearly defined, the codes were considered as missing data.

Agreement was considered a binary outcome. Agreement occurred when the most important condition identified by the patient matched one of the two conditions given by the GP. The independent variables of number of years of practice, number of years of follow-up by the GP, the patient's age, and the number of visits to the GP in the last 12 months were considered as continuous variables. All other variables were considered as categorical variables. The different numbers of chronic conditions were stratified into three groups: " 3 ", " 4 or 5 ", and "6 or more" chronic conditions. This categorization was made according to the levels of multimorbidity defined by Kadam et al. [17]

\section{Study size}

In all, 888 patients were included in the cross-sectional study. After discarding patients with missing values for the matching variables, 841 patients remained. From these patients, we excluded those with ambiguous responses, i.e. responses that could not easily be assigned to one category. As a result, 579 and 593 were retained for the main and sensitivity analyses, respectively. We eliminated patients with incomplete data for any of the independent variables considered. Finally, 572 and 585 patients (64.4 and $65.9 \%$ of the original sample) were included in the main and sensitivity analyses, respectively. Patients discarded due to missing data were not different from the patients included in the main analysis in terms of sex (chi $\left.{ }^{2}, 1 \mathrm{df}, p=0.05\right)$, number of chronic conditions ( $\left.\mathrm{chi}^{2}, 2 \mathrm{df}, p=0.45\right)$, level of education $\left(\mathrm{chi}^{2}\right.$, $6 \mathrm{df}, p=0.25)$, number of MDs involved in patient's treatment ( $\left.\mathrm{chi}^{2}, 3 \mathrm{df}, p=0.24\right)$, number of GP visits in last 12 months (t-test, $p=0.86$ ). They were slightly older than patients included (mean age (SD): 74.3 (11.5) versus $72.2(12.2)$, t-test $p<0.05)$.

\section{Analyses}

For both analyses, we examined whether one of the two conditions identified by the GP matched the most important condition identified by the patient. For the main analysis, agreement was evaluated with regards to 46 groups of conditions (Additional file 1: Table S1), whereas for the sensitivity analysis this was done with regards to 15 chapters of the ICPC-2 classification.

\section{Statistical methods}

We conducted bivariate and multivariate logistic regressions to evaluate the associations between GP-related 
and patient-related variables with the agreement variable and to evaluate any potential clustering effects around GPs' variables.

\section{Results}

Agreements between the most important health condition for patients and those perceived to be the most important to patients by GPs are reported in Table 1 . In the main analysis, agreement occurred in $54.9 \%$ of cases $(N=314)$. For the sensitivity analysis, agreement occurred in $63.8 \%$ of the cases $(N=373)$ (Table 1$)$. No clustering effects around GPs' variables were detected.

In the main model based on the classification of items in 46 groups, only the number of chronic conditions was significantly associated with the outcome both in univariate (Table 2) and multivariate analyses (Table 3).

More precisely, agreement between GPs and patients decreased as the number of chronic conditions increased. Compared to " 3 " chronic conditions, the odds ratios (95\% confidence interval, CI) for agreement between when there were " 4 or 5 " and " 6 or more" chronic conditions were 0.53 (95\% CI, 0.31-0.89) and 0.59 (95\% CI, 0.34-1), respectively. Thus, there was only a statistically significant difference in agreement when the " 4 or 5 " conditions group was compared to the " 3 " chronic conditions group. No cluster effects were observed around any of the GPs, and the rates of agreement were similar for all the GPs enrolled. Rates of agreement were $65.6,50.8$, and $55.1 \%$ for the " 3 ", " 4 or 5 ", and " 6 or more" chronic conditions groups, respectively (Table 4).

The groups of conditions most frequently mentioned by patients as the most important conditions included Musculoskeletal conditions and pain and Spine conditions/back pain (groups 18, 19). For these two groups of conditions, rates of agreement between GPs and patients were 67.8 and 42.9\%, respectively (Additional file 2: Table S2 a). Unfortunately for this analysis, the limited number of observations in some groups of conditions limited the interpretation of results.
The sensitivity analysis conducted on broader groups of conditions relating to chapters if the ICPC-2 showed similar results. Compared to " 3 " chronic conditions, the odds ratio for agreement between when there were " 4 or 5 " and " 6 or more" chronic conditions were 0.6 (95\% CI, $0.34-1.01$ ) and 0.64 (95\% CI, 0.36-1.09), respectively (Additional file 2: Table S2 b).

\section{Discussion}

When chronic conditions were classified into 46 categories, GPs were able to identify the condition most important to their patients in $54.9 \%$ of cases. The rate of agreement was higher $(63.8 \%)$ when looking for agreement with items grouped in the chapters of the ICPC-2 classification. Differences between the perspectives of GPs and patients have previously been shown for such aspects of disease management as the cure or relief of symptoms [18], disease severity [19], and the level of suffering [20]. In the present study, back pain and musculoskeletal conditions, often symptomatic, were the conditions most often ranked first by patients. However, agreement with GPs on these conditions was contrasted, being higher than average for musculoskeletal conditions but lower for spine and back syndromes. Any interpretation of these analyses is limited by the reduced number of conditions per group. Nevertheless, these results seem to confirm that pain and symptomatic chronic conditions are patients' first priorities, whereas GPs' priorities may be more prognosis-based [7].

The only variable associated with a difference in the rates of agreement between GPs and patients was the number of chronic conditions. This was expected because the probability of patient-GP agreement is higher in patients with fewer health conditions $[5,6]$. In the present study, however, the rate of agreement for patients suffering from 4 or 5 chronic conditions was lower than for patients with 3 chronic conditions, but the rate did not decrease further for patients suffering from 6 or more. This suggests that patient-GP agreement may depend somewhat on factors

Table 1 Patient-GP rates of agreement on the condition ranked as most important to the patient (data collection JanuarySeptember 2015)

\begin{tabular}{|c|c|c|c|c|c|}
\hline \multirow[b]{2}{*}{ Agreement } & \multirow[b]{2}{*}{$\begin{array}{l}\text { The condition that the GP thinks is most important } \\
\text { to the patient belongs to the same group/chapter } \\
\text { as the condition considered most important by the } \\
\text { patient. }\end{array}$} & \multicolumn{2}{|c|}{$\begin{array}{l}46 \text { groups of conditions }(N=572) \\
N(\%)\end{array}$} & \multicolumn{2}{|c|}{$\begin{array}{l}15 \text { chapters of the ICPC- } 2(N=585) \\
N(\%)\end{array}$} \\
\hline & & 217 (37.9) & \multirow[t]{2}{*}{$314(54.9)$} & $260(44.4)$ & \multirow[t]{3}{*}{$373(63.8)$} \\
\hline & $\begin{array}{l}\text { The condition that the GP thinks is the second most } \\
\text { important to the patient belongs to the same group/ } \\
\text { chapter as the condition considered most important } \\
\text { by the patient. }\end{array}$ & 97 (17) & & 113 (19.3) & \\
\hline Disagreement & $\begin{array}{l}\text { The conditions that the GP thinks are most important to } \\
\text { the patient do not belong to the same group/chapter } \\
\text { as the condition considered most important by the patient. }\end{array}$ & \multicolumn{2}{|l|}{$258(45.1)$} & $212(36.2)$ & \\
\hline
\end{tabular}


Table 2 Bivariate analyses of all the variables considered in the study (Data collection January-September 2015)

\begin{tabular}{|c|c|c|c|c|c|c|c|}
\hline & & \multicolumn{3}{|c|}{46 groups of conditions $(N=572)$} & \multicolumn{3}{|c|}{15 chapters of the ICPC-2 $(N=585)$} \\
\hline & & No & Yes & $p$-value & No & Yes & $p$-value \\
\hline \multicolumn{8}{|l|}{ Patient-related variables } \\
\hline \multirow[t]{3}{*}{ No. of chronic conditions ${ }^{+}$} & $3, N(\%)$ & $31(12)$ & $59(18.8)$ & 0.06 & $26(12.3)$ & $66(17.7)$ & 0.20 \\
\hline & 4 or 5 & $121(46.9)$ & $125(39.8)$ & & $98(46.2)$ & $154(41.3)$ & \\
\hline & 6 or more & $106(41.1)$ & $130(41.4)$ & & $88(41.5)$ & $153(41)$ & \\
\hline $\mathrm{Age}^{++}$ & Mean, years (SD) & $73.1(12.1)$ & $71.5(12.3)$ & 0.11 & $73(11.5)$ & $71.9(12.7)$ & 0.28 \\
\hline \multirow[t]{2}{*}{$\operatorname{Sex}^{+}$} & Male, N (\%) & $117(45.3)$ & $144(45.9)$ & 0.93 & $101(47.6)$ & 171 (45.8) & 0.73 \\
\hline & Female & $141(54.7)$ & $170(54.1)$ & & $111(52.4)$ & $202(54.2)$ & \\
\hline \multirow[t]{7}{*}{ Level of education+ } & Primary school/no diploma, N (\%) & $43(16.7)$ & $42(13.4)$ & 0.28 & $36(17)$ & $51(13.7)$ & 0.82 \\
\hline & Secondary school & $20(7.8)$ & $24(7.6)$ & & $16(7.5)$ & $28(7.5)$ & \\
\hline & Practical professional training & $61(23.6)$ & $86(27.4)$ & & $56(26.4)$ & $94(25.2)$ & \\
\hline & High school or equivalent & $33(12.8)$ & $33(10.5)$ & & $23(10.8)$ & $45(12.1)$ & \\
\hline & Vocational school or training & $58(22.5)$ & $92(29.3)$ & & $51(24.1)$ & $105(28.2)$ & \\
\hline & Non-university higher education & $26(10.1)$ & $24(7.6)$ & & $17(8)$ & $33(8.8)$ & \\
\hline & University & $17(6.6)$ & $13(4.1)$ & & $13(6.1)$ & $17(4.6)$ & \\
\hline \multirow{4}{*}{$\begin{array}{l}\text { No. of MDs involved in patient's } \\
\text { treatment }^{+}\end{array}$} & 1, N (\%) & $73(28.3)$ & $81(25.8)$ & 0.81 & $58(27.4)$ & $98(26.3)$ & 0.75 \\
\hline & 2 & $76(29.5)$ & $89(28.3)$ & & $57(26.9)$ & $112(30)$ & \\
\hline & 3 & $54(20.9)$ & $75(23.9)$ & & $48(22.6)$ & $88(23.6)$ & \\
\hline & 4 or more & $55(21.3)$ & $69(22)$ & & $49(23.1)$ & $75(20.1)$ & \\
\hline No. of GP visits in last 12 months ${ }^{++}$ & Mean, N (SD) & $12.9(8.9)$ & $13(8.7)$ & 0.87 & $13.2(9.3)$ & $13.2(9.2)$ & 0.95 \\
\hline No. of years followed by $\mathrm{GP}^{++}$ & Mean, years (SD) & $10.9(7.9)$ & $11.2(8.5)$ & 0.59 & $10.4(7.9)$ & $11.5(8.4)$ & 0.13 \\
\hline \multicolumn{8}{|l|}{ GP-related variables } \\
\hline \multirow[t]{2}{*}{ Sext } & Male, N (\%) & $196(76)$ & $220(70.1)$ & 0.13 & $152(71.7)$ & $271(72.7)$ & 0.85 \\
\hline & Female & $62(24)$ & $94(29.9)$ & & $60(28.3)$ & $102(27.3)$ & \\
\hline No. of years of practice++ & Mean, years (SD) & $17.3(9.6)$ & $18(9.6)$ & 0.42 & $16.5(9.5)$ & $18.2(9.5)$ & 0.03 \\
\hline \multirow[t]{5}{*}{ No. of consultations per week+ } & $\leq 30 \mathrm{~N}(\%)$ & $2(0.8)$ & $3(1)$ & 0.82 & $1(0.5)$ & $4(1.1)$ & 0.59 \\
\hline & ]30-70] & $72(27.9)$ & $84(26.8)$ & & $60(28.3)$ & $96(25.7)$ & \\
\hline & ]70-110] & $117(45.3)$ & $148(47.1)$ & & $100(47.2)$ & $176(47.2)$ & \\
\hline & ]110-170] & $59(22.9)$ & $64(20.4)$ & & $46(21.7)$ & $79(21.2)$ & \\
\hline & $>170$ & $8(3.1)$ & $15(4.8)$ & & $5(2.4)$ & $18(4.8)$ & \\
\hline \multirow[t]{3}{*}{ Practice location+ } & Urban, N (\%) & $109(42.2)$ & $136(43.3)$ & 0.97 & $92(43.4)$ & $157(42.1)$ & 0.86 \\
\hline & Suburban & $108(41.9)$ & $129(41.1)$ & & $85(40.1)$ & $158(42.4)$ & \\
\hline & Rural & $41(15.9)$ & 49 (15.6) & & $35(16.5)$ & $58(15.5)$ & \\
\hline \multirow{2}{*}{$\begin{array}{l}\text { Sub-specialization in psychosocial \& } \\
\text { psychosomatic medicine }\end{array}$} & No, N (\%) & $236(91.5)$ & $294(93.6)$ & 0.34 & 198 (93.4) & 347 (93) & 1.00 \\
\hline & Yes & $22(8.5)$ & $20(6.4)$ & & $14(6.6)$ & $26(7)$ & \\
\hline
\end{tabular}

Agreement is positive if one of the two conditions the GP considered as important to the patient belongs to the same category as the condition considered most important by the patient. Otherwise, it is negative. Difference between groups tested using: + Fisher test; ++ t-test; practice location was evaluated using zip codes and data obtained from the Swiss Federal Statistical Office

other than those evaluated in this study. Similarly to our results, a previous study showed that a GP's knowledge of a patient's health was not influenced by the patient's age, level of education, number of consultations in the previous 6 months or the duration of the patient-GP relationship [6]. Although the present study did not report the GP's sex, this did not seem to be associated with agreement on important health conditions [5].
Our study failed to show any positive associations between the rate of patient-GP agreement and a subspecialization in psychosocial or psychosomatic medicine, a longer follow-up, or more frequent appointments. This was despite our anticipation that these factors would have given GPs greater knowledge of their patients. An alternative explanation could be that patients with more frequent appointments and longer follow-up 
Table 3 - Multivariate analyses of all the variables considered in the study (Data collection January-September 2015)

\begin{tabular}{|c|c|c|c|c|c|}
\hline & & \multicolumn{2}{|l|}{$\begin{array}{l}46 \text { groups of } \\
\text { conditions }(N=572)\end{array}$} & \multicolumn{2}{|l|}{$\begin{array}{l}15 \text { chapters of } \\
\text { ICPC-2 }(\mathrm{N}=585)\end{array}$} \\
\hline & & Odds ratio $(95 \% \mathrm{Cl})$ & $p$-value & Odds ratio $(95 \% \mathrm{Cl})$ & $p$-value \\
\hline \multicolumn{6}{|l|}{ Patient-related variables } \\
\hline \multirow[t]{3}{*}{ No. of chronic conditions } & 3 (reference) & & & & \\
\hline & 4 or 5 & $0.53(0.31 ; 0.89)$ & 0.02 & $0.6(0.34 ; 1.01)$ & 0.06 \\
\hline & 6 or more & $0.59(0.34 ; 1)$ & 0.05 & $0.64(0.36 ; 1.09)$ & 0.11 \\
\hline Age & per year & $0.99(0.98 ; 1.01)$ & 0.22 & $0.99(0.98 ; 1.01)$ & 0.32 \\
\hline \multirow[t]{2}{*}{ Sex } & Male (reference) & & & & \\
\hline & Female & $0.98(0.69 ; 1.41)$ & 0.92 & $1.11(0.77 ; 1.59)$ & 0.59 \\
\hline \multirow[t]{7}{*}{ Level of education } & Primary school/ no diploma (reference) & & & & \\
\hline & Secondary school & $1.17(0.55 ; 2.51)$ & 0.68 & $1.18(0.55 ; 2.58)$ & 0.68 \\
\hline & Practical professional training & $1.53(0.87 ; 2.69)$ & 0.14 & $1.16(0.66 ; 2.03)$ & 0.60 \\
\hline & High school or equivalent & $1.05(0.54 ; 2.05)$ & 0.89 & $1.33(0.68 ; 2.65)$ & 0.41 \\
\hline & Vocational school or training & $1.66(0.94 ; 2.94)$ & 0.08 & $1.45(0.82 ; 2.57)$ & 0.20 \\
\hline & Non-university higher education & $0.89(0.42 ; 1.89)$ & 0.76 & $1.28(0.59 ; 2.8)$ & 0.54 \\
\hline & University & $0.79(0.32 ; 1.92)$ & 0.60 & $0.91(0.37 ; 2.25)$ & 0.84 \\
\hline \multirow[t]{4}{*}{ No. of MDs involved in patient's treatment } & 1 (reference) & & & & \\
\hline & 2 & $1.05(0.66 ; 1.67)$ & 0.84 & $1.13(0.7 ; 1.82)$ & 0.62 \\
\hline & 3 & $1.22(0.74 ; 2.01)$ & 0.44 & $1.07(0.64 ; 1.78)$ & 0.80 \\
\hline & 4 or more & $1.11(0.67 ; 1.84)$ & 0.69 & $0.87(0.52 ; 1.45)$ & 0.59 \\
\hline No. of GP visits in last 12 months & per unit & $0(0 ; 0)$ & & $0(0 ; 0)$ & \\
\hline No. of years followed by GP & per year & $1(0.97 ; 1.03)$ & 0.92 & $1(0.97 ; 1.03)$ & 0.95 \\
\hline \multicolumn{6}{|l|}{ GP-related variables } \\
\hline \multirow[t]{2}{*}{ Sex } & Male (reference) & & & & \\
\hline & Female & $1.49(0.96 ; 2.33)$ & 0.08 & $0.99(0.64 ; 1.54)$ & 0.96 \\
\hline No. of years of practice & per year & $1.01(0.99 ; 1.04)$ & 0.39 & $1.02(0.99 ; 1.05)$ & 0.20 \\
\hline \multirow[t]{5}{*}{ No. of consultations per week } & $\leq 30$ (reference) & & & & \\
\hline & ]30-70] & $0.82(0.1 ; 5.49)$ & 0.83 & $0.43(0.02 ; 3.26)$ & 0.47 \\
\hline & ]70-110] & $1.01(0.12 ; 7.01)$ & 0.99 & $0.43(0.02 ; 3.36)$ & 0.48 \\
\hline & ]110-170] & $0.92(0.11 ; 6.56)$ & 0.94 & $0.42(0.02 ; 3.38)$ & 0.47 \\
\hline & $>170$ & $1.36(0.14 ; 11.61)$ & 0.78 & $0.78(0.03 ; 8.1)$ & 0.85 \\
\hline \multirow[t]{3}{*}{ Practice location } & Urban (reference) & & & & \\
\hline & Suburban & $0.92(0.62 ; 1.36)$ & 0.68 & $1.08(0.73 ; 1.61)$ & 0.70 \\
\hline & Rural & $0.92(0.54 ; 1.59)$ & 0.77 & $1(0.58 ; 1.73)$ & 0.99 \\
\hline \multirow{2}{*}{$\begin{array}{l}\text { Sub-specialization in psychosocial \& } \\
\text { psychosomatic medicine }\end{array}$} & No (reference) & & & & \\
\hline & Yes & $0.76(0.38 ; 1.49)$ & 0.42 & $1.08(0.53 ; 2.26)$ & 0.84 \\
\hline
\end{tabular}

Table 4 Rates of agreement in relation to the number of chronic conditions, split into 46 groups and 15 chapters of the ICPC-2

\begin{tabular}{|c|c|c|c|c|c|c|c|c|}
\hline \multirow{3}{*}{$\begin{array}{l}\text { Number of chronic conditions } \\
3\end{array}$} & \multicolumn{4}{|c|}{$\begin{array}{l}\text { Agreement on one of the } 46 \text { groups of conditions - } \\
\text { main analysis }(N=572)\end{array}$} & \multicolumn{4}{|c|}{$\begin{array}{l}\text { Agreement on one of the } 15 \text { chapters of the ICPC-2 classification } \\
\text { - sensitivity analysis }(N=585)\end{array}$} \\
\hline & \multicolumn{2}{|c|}{ No agreement } & \multicolumn{2}{|c|}{ Agreement } & \multicolumn{2}{|c|}{ No agreement } & \multicolumn{2}{|c|}{ Agreement } \\
\hline & 31 & $34.4 \%$ & 59 & $65.6 \%$ & 26 & $28.3 \%$ & 66 & $71.7 \%$ \\
\hline 4 or 5 & 121 & $49.2 \%$ & 125 & $50.8 \%$ & 98 & $38.9 \%$ & 154 & $61.1 \%$ \\
\hline 6 or more & 106 & $44.9 \%$ & 130 & $55.1 \%$ & 88 & $36.5 \%$ & 153 & $63.5 \%$ \\
\hline
\end{tabular}


suffer from more chronic conditions and are more complicated for GPs to handle.

Our study has some limitations. To identify the health conditions most important to patients, we asked them which of their chronic health problems they would like to heal first and second, given the opportunity. Although admittedly the question was not formally validated, both GPs and patients deemed the question appropriate during a pilot phase But, it slightly differed from the question asked to GPs. Using the term "healing" with patients might have encouraged them to list their most symptomatic conditions. But, as previous studies showed that in any case patients with multimorbidity generally prioritized the treatment of symptomatic diseases over asymptomatic diseases $[5,21,22]$ we do not believe that this could have biased our study's outcome to a great extent. We also did not evaluate the importance of certain other factors previously identified as influencing patients' prioritization of health problems, such as emotional disorders interfering with day-to-day activities and perceived unfavorable diagnoses [9]. And we did not include detailed information about duration, severity, treatment side effects, or functional impairments linked to each condition. Finally, patients' priorities regarding multiple chronic conditions are likely to change over time in response to factors such as a worsening of one of the conditions [23], the negative effects of a treatment [24], or contact with health professionals [23, 24] and should be re-examined whenever the patient's clinical status changes [4].

An alternative explanation for the absence of positive associations between any of the patient or GP variables considered and the rate of patient-GP agreement could be related to a lack of patient-centered care. Indeed, patient-centered care could be a more holistic or better overall way of treating patients with multimorbidity; one more centered on general priorities such as staying alive, maintaining independence, reducing/eliminating pain, and reducing/eliminating symptoms rather than on treating specific health conditions or diseases. A study evaluating patients' prioritization of these outcomes reported that $76 \%$ ranked maintaining independence as the most important factor; this was followed by the relief of symptoms [25]. In another study of patients with multimorbidity, of those who ranked staying alive as their primary concern (11\%), 66\% ranked maintaining independence as their secondary concern [25].

\section{Conclusion}

The present national, cross-sectional study of multimorbidity in Switzerland is one of the first evaluations of GPs' ability to identify the chronic health condition that their patients consider most important. We showed that in more than half of the cases examined, GPs were able to identify this condition, as referenced in a classification of 46 groups of conditions. This rose to about two thirds of cases when the conditions were grouped by chapter in the ICPC-2 classification. In view of these results, it seems crucial that GPs evaluate, which conditions are most important to their patients and subsequently adapt treatment targets and priorities accordingly. Good communication and patient-provider agreement would seem to be essential in this regard. Further research is needed to understand the factors underlying rates of agreement between GPs and patients, as well as which aspects-specific health conditions and symptoms or a more comprehensive or alternately holistic treatment method-should be at the heart of the patient-centered care for patients with multimorbidity.

\section{Additional files}

Additional file 1: Table S1. Classification of items of the ICPC-2 into 46 categories adapted from van den Bussche et al. (DOCX 25 kb)

Additional file 2: Table S2. a Agreement as per the 46 groups of conditions ( $N=572$ ). Table S2. b Agreement as per the chapters of the ICPC-2 classification ( $N=585)$. (DOCX $20 \mathrm{~kb})$

\section{Abbreviation}

GP: General practitioners

\section{Acknowledgements}

We would like to thank the 100 GPs who participated in this study for their valuable feedback, as well as all the participating patients.

\section{Funding}

The Swiss University Conference has granted financial support to reinforce teaching and research on primary care in Switzerland. Within this framework, the country's five institutes for Family Medicine (Basel, Bern, Geneva, Zürich, and Lausanne) collaborate as the Swiss Academy for Family Medicine (SAFMED). Part of the funding must be used for national research projects on family medicine.

\section{Availability of data and materials}

All data generated or analysed during this study are included in this published article. Original datas are available at the Institute of Family Medicine, University of Lausanne.

\section{Authors' contributions}

$A D L, A A N, N S, P B, B B, D W, R T, A Z, D M H, T R$, and $L H$ developed the protocol and defined the study's objectives. ADL, AAN, NS, PB, BB, DW, LH, RT, DMH, and $A Z$ defined the list of variables to be collected. LH, SZ, SNJ constructed the item classifications. $\mathrm{LH}, \mathrm{AZ}, \mathrm{DMH}$, and SS recruited the participating GPS. $A D L$, JP conducted the statistical analyses. All authors contributed to the development and revision of the manuscript and to the decision to submit it for publication. All authors read and approved the final manuscript.

\section{Ethics approval and consent to participate}

The study protocol ( $n^{\circ} 314 / 15$ ) was approved by the Human Research Ethics Committee of the Canton Vaud. GPs informed identified patients about the study, and patients provided written informed consent. Only anonymous dates were transmitted to the research team.

Competing interests

The authors declare that they have no competing interests. 


\section{Publisher's Note}

Springer Nature remains neutral with regard to jurisdictional claims in published maps and institutional affiliations.

\section{Author details}

'Institute of Family Medicine, University of Lausanne, 44 rue du Bugnon, 1011 Lausanne, Switzerland. ${ }^{2}$ Institute of Social and Preventive Medicine, Lausanne University Hospital, Lausanne, Switzerland. ${ }^{3}$ Department of Ambulatory Care and Community Medicine, Lausanne University Hospital, Lausanne, Switzerland. ${ }^{4}$ Institute of Primary Care, University of Zurich, Zurich, Switzerland. ${ }^{5}$ Institute of Primary Health Care (BIHAM), University of Berne, Berne, Switzerland. ${ }^{6}$ Centre for Primary Health Care, University of Basel, Basel, Switzerland. 'Primary Care Unit, Faculty of Medicine, University of Geneva, Geneva, Switzerland

Received: 11 August 2017 Accepted: 1 May 2018

Published online: 17 May 2018

\section{References}

1. Van den Akker MBF, Knottnerus JA. Comorbidity or multimorbidity: what's in a name? A review of literature. Eur J Gen Pract. 1996;2(2):65-70.

2. Starfield B, Wray C, Hess K, Gross R, Birk PS, D'Lugoff BC. The influence of patient-practitioner agreement on outcome of care. Am J Public Health. 1981;71(2):127-31.

3. Kerr EA, Heisler M, Krein SL, Kabeto M, Langa KM, Weir D, et al. Beyond comorbidity counts: how do comorbidity type and severity influence diabetes patients' treatment priorities and self-management? J Gen Intern Med. 2007;22(12):1635-40.

4. Laiteerapong $\mathrm{N}$, Huang ES, Chin MH. Prioritization of care in adults with diabetes and comorbidity. Ann N Y Acad Sci. 2011;1243:69-87.

5. Zulman DM, Kerr EA, Hofer TP, Heisler M, Zikmund-Fisher BJ. Patientprovider concordance in the prioritization of health conditions among hypertensive diabetes patients. J Gen Intern Med. 2010;25(5):408-14

6. Stewart MA, McWhinney IR, Buck CW. The doctor/patient relationship and its effect upon outcome. J R Coll Gen Pract. 1979:29(199):77-81.

7. Neuner-Jehle S, Zechmann S, Grundmann Maissen D, Rosemann T, Senn O. Patient-provider concordance in the perception of illness and disease: a cross-sectional study among multimorbid patients and their general practitioners in Switzerland. Patient Prefer Adherence. 2017:11:1451-8.

8. Voigt I, Wrede J, Diederichs-Egidi H, Dierks ML, Junius-Walker U. Priority setting in general practice: health priorities of older patients differ from treatment priorities of their physicians. Croat Med J. 2010;51(6):483-92.

9. Junius-Walker U, Stolberg D, Steinke $P$, Theile G, Hummers-Pradier E, Health DML. Treatment priorities of older patients and their general practitioners: a cross-sectional study. Qual Prim Care. 2011;19(2):67-76.

10. Fredericksen RJ, Edwards TC, Merlin JS, Gibbons LE, Rao D, Batey DS, et al. Patient and provider priorities for self-reported domains of HIV clinical care. AIDS Care. 2015;27(10):1255-64.

11. Deruaz-Luyet A, N'Goran AA, Tandjung R, Frey P, Zeller A, Haller DM, et al. Multimorbidity in primary care: protocol of a national cross-sectional study in Switzerland. BMJ Open. 2015;5(10):e009165.

12. Deruaz-Luyet A, N'Goran AA, Senn N, Bodenmann P, Pasquier J, Widme $D$, et al. Multimorbidity and patterns of chronic conditions in a primary care population in Switzerland: a cross-sectional study. BMJ Open. 2017; 7(6):e013664.

13. N'Goran AA, Blaser J, Deruaz-Luyet A, Senn N, Frey P, Haller DM, et al. From chronic conditions to relevance in multimorbidity: a four-step study in family medicine. Family Practice. 2016:cmw030.

14. Hofmans-Okkes IM, Lamberts $H$. The international classification of primary care (ICPC): new applications in research and computer-based patient records in family practice. Fam Pract. 1996;13(3):294-302.

15. van den Bussche H, Koller D, Kolonko T, Hansen H, Wegscheider K, Glaeske $G$, et al. Which chronic diseases and disease combinations are specific to multimorbidity in the elderly? Results of a claims data based cross-sectional study in Germany. BMC Public Health. 2011;11:101.

16. Bentsen BG. International classification of primary care. Scand J Prim Health Care 1986:4(1):43-50.

17. Kadam UT, Croft PR, North Staffordshire GPCG. Clinical multimorbidity and physical function in older adults: a record and health status linkage study in general practice. Fam Pract. 2007;24(5):412-9.
18. Anden A, Andre M, Rudebeck CE. What happened? GPs' perceptions of consultation outcomes and a comparison with the experiences of their patients. Eur J Gen Pract. 2010;16(2):80-4.

19. Kroenke K, Wyrwich KW, Tierney WM, Babu AN, Wolinsky FD. Physicianestimated disease severity in patients with chronic heart or lung disease: a cross-sectional analysis. Health Qual Life Outcomes. 2006:4:60.

20. Lesho E, Foster L, Wang Z, Sarmiento D, Dennison S, Vahey MT, et al. The accuracy of physicians' perceptions of patients' suffering: findings from two teaching hospitals. Acad Med. 2009;84(5):636-42.

21. Fried LP, Bandeen-Roche K, Kasper JD, Guralnik JM. Association of comorbidity with disability in older women: the Women's health and aging study. J Clin Epidemiol. 1999;52(1):27-37.

22. Fultz NH, Ofstedal MB, Herzog AR, Wallace RB. Additive and interactive effects of comorbid physical and mental conditions on functional health. J Aging Health. 2003:15(3):465-81.

23. Cheraghi-Sohi S, Bower P, Kennedy A, Morden A, Rogers A, Richardson J, et al. Patient priorities in osteoarthritis and comorbid conditions: a secondary analysis of qualitative data. Arthritis Care Res (Hoboken). 2013; 65(6):920-7.

24. Morris RL, Sanders C, Kennedy AP, Rogers A. Shifting priorities in multimorbidity: a longitudinal qualitative study of patient's prioritization of multiple conditions. Chronic IIIn. 2011:7(2):147-61.

25. Fried TR, Tinetti ME, lannone L, O'Leary JR, Towle V, Van Ness PH. Health outcome prioritization as a tool for decision making among older persons with multiple chronic conditions. Arch Intern Med. 2011;171(20):1854-6.

\section{Ready to submit your research? Choose BMC and benefit from:}

- fast, convenient online submission

- thorough peer review by experienced researchers in your field

- rapid publication on acceptance

- support for research data, including large and complex data types

- gold Open Access which fosters wider collaboration and increased citations

- maximum visibility for your research: over $100 \mathrm{M}$ website views per year

At BMC, research is always in progress.

Learn more biomedcentral.com/submissions 